\title{
Editorial to themed issue: Recent advances in cardiovascular pharmacokinetic-pharmacodynamic modeling and simulation
}

\author{
Peter Bonate ${ }^{1}$
}

Received: 9 April 2018 / Accepted: 23 April 2018/Published online: 2 May 2018

(C) Springer Science+Business Media, LLC, part of Springer Nature 2018

In 2012 we started a new idea at the Journal-a themed disease state issue. Our first issue in 2013 was Translational Modeling in Neuroscience, followed in 2014 by Modeling the Immune Response to Infection, and last year with a Special Focus on Infectious Disease. This year our theme is cardiovascular disease and this issue is entitled Recent Advances in Cardiovascular Pharmacokinetic-Pharmacodynamic Modeling and Simulation.

Let's face it-cardiovascular (CV) drugs are not sexy. The amount of research dollars spent in this therapeutic area has been declining for over 2 decades leading the American College of Cardiology (ACC) to question whether the research area is dead [1]. And it's easy to see why. In 2016 there were no CV drugs among the Top 10 in terms of sheer sales and there was only 1 drug in the Top 15 (\#11: Xarelto $^{\circledR}$, rivaroxaban, $\$ 5.4$ billion) [2]. The costs of conducting $\mathrm{CV}$ drug trials are among the highest because of the large number of patients needed to demonstrate adequate safety and efficacy. Further, clinical outcome studies often take years to complete. But in terms in number of total number of prescriptions written, cardiovascular drugs fill out the top ten: lisinopril (\#1), atorvastatin (\#3), simvastatin (\#5), amlodipline (\#7), metoprolol (\#8) [3]. CV drugs clearly represent a major prescribing category for physicians but there is a disconnect between prescribing needs and research and development.

In the editorial written by the ACC they presented some strategies to advance $\mathrm{CV}$ drug development. These included:

- Reduce operating costs of clinical trials;

- Increase focus on practical, streamlined trials;

- Leverage Phase 2 trials to inform Phase 3;

- Continue to strengthen the science; and

- Use novel clinical trial designs.

Peter Bonate

Peter.bonate@astellas.com

Astellas, 1 Astellas Way, Northbrook, IL 60062, USA
Modeling and simulation (M\&S) can support all these efforts. Quantitative systems pharmacology (QSP). Physiological-based pharmacokinetic-pharmacodynamic modeling. Traditional pharmacokinetic-pharmacodynamic (PKPD) modeling. Discrete event simulation. All these methods can be used to increase the efficiency of drug development, not just $\mathrm{CV}$ drug development, although sadly, in that editorial by the ACC, the only one of these methods mentioned as a strategy to improve CV drug development was population pharmacokinetic (PopPK) modeling.

As a discipline we need to do better at promoting the value of $\mathrm{M} \& S$ to drug development, beyond PopPK. Ironically, CV drugs may be the best therapeutic class to demonstrate the value of $M \& S$ because of the availability of readily collected biomarkers and surrogate markers. In that spirit we have collected in this issue a variety of articles ranging from preclinical to clinical, from traditional PKPD to QSP, from research articles to tutorials, demonstrating the value of M\&S in CV drug development. Although not meant to be comprehensive, the breadth of articles can serve as a basis for those wishing to promote pharmacometrics in this therapeutic area. The editors want to thank all the contributors for their hard work and their patience as we worked to collect all these excellent articles.

\section{References}

1. Fordyce CB, Roe MT, Ahmad T, Libby P et al (2015) Cardiovascular drug development: is it dead or just hibernating? J Am Coll Cardiol 65:1567-1582

2. The Top 15 best-selling drugs of 2016. Genetic engineering and biotech news, 6 March 2017. https://www.genengnews.com/thelists/the-top-15-best-selling-drugs-of-2016/77900868. Accessed 9 April 2018

3. Top 200 drugs of 2018. ClinCalc drug stats database. http:// clincalc.com/DrugStats/. Accessed 9 April 2018 